\title{
Impact Des Structures Fiscales Sur La Croissance Economique Au Congo-Brazzaville
}

\author{
Ferdinand Moussavou \\ Faculté des Sciences Economiques, \\ Université Marien Ngouabi, Brazzaville, Congo
}

Doi: 10.19044/esj.2017.v13n34p163 URL:http://dx.doi.org/10.19044/esj.2017.v13n34p163

\begin{abstract}
This article proposes to make from Congolese data an empirical evaluation of the impact of tax structures on economic growth. It is based on the effects of state transfers to businesses and on the distinction between personal income taxes, corporate taxes and taxes on goods and services. The results obtained from Dickey and Fuller's model show that, in the long term, personal income taxes, taxes on goods and services, total state revenues, state transfers to enterprises and gross fixed capital formation affect economic growth. In the short term, corporate taxes, revenues and transfers from the state to companies exert an influence on this growth. On the other hand, long-term and short-term results show that government revenues and transfers affect economic growth. These results made it possible to identify the limits of the economic policies implemented in Congo-Brazzaville.
\end{abstract}

Keywords: Tax structures, economic growth, transfers from the state

Résumé

Cet article se propose de réaliser à partir des données congolaises, une évaluation empirique de l'impact des structures fiscales sur la croissance économique. Il s'appuie sur les effets des transferts de l'Etat aux entreprises et sur la distinction entre les impôts sur les revenus des personnes physiques, les impôts sur les sociétés et impôts sur les biens et services. Les résultats obtenus à partir du modèle de Dickey et Fuller montrent qu'à long terme, les impôts sur les revenus des personnes physiques, les impôts sur les biens et services, les recettes totales de l'Etat, les transferts de l'Etat aux entreprises et la formation brute de capital fixe impactent la croissance économique. A court terme, les impôts sur les sociétés, les recettes et les transferts de l'Etat aux entreprises exercent une influence sur cette croissance. En revanche à long terme et court terme, les résultats montrent que les recettes et les transferts de l'Etat affectent la croissance économique. Ces résultats ont 
permis de dégager les limites des politiques économiques mises en œuvre au Congo-Brazzaville.

Mots clés: Structures fiscales, croissance économique, transferts de l’Etat.

\section{Introduction}

Les économistes (Smith, 1776 ; Solow, 1956; Feldstein, 1974), soutiennent que les structures fiscales, c'est-à-dire les impôts, leur niveau et la façon dont ils sont combinés (OCDE, 2009, 146) influent sur les décisions des individus concernant l'épargne, le travail, les décisions des entreprises en matière de production, de création d'emplois, d'investissement et d'innovation. Leurs effets sur la croissance peuvent être positifs ou négatifs.

De nombreux travaux ont démontré l'impact positif des structures fiscales sur la croissance économique. Corlett et Hague (1953) et Heady (1987) ont par exemple, analysé l'incidence des structures des impôts sur la croissance économique. Pour ces auteurs la structure de consommation influe positivement sur l'offre de main-d'œuvre.

Cependant, Poterba (1989), Levine et Renelt (1992), Chambas (1994) et Engen et Skinner (1996) ont aboutit à des résultats contraires à savoir que les structures fiscales entretiennent des relations négatives sur la croissance économique. Poterba (1989) étudient les effets de l'impôt sur les gains en capital sur l'investissement. Il confirme que l'impôt sur les gains en capital bloque l'investissement et empêche une réallocation efficace du capital. Levine et Renelt (1992) explorent les effets des impôts sur les sociétés, des impôts sur les personnes physiques et des prélèvements sociaux sur la croissance. Ils trouvent que ces variables réduisent le taux de croissance. Dans la même lignée, Chambas (1994) et Engen et Skinner (1996) confirment ce résultat lorsqu'ils étudient l'incidence des impôts directs sur l'économie des nations. Ils concluent que les taux d'imposition ont des effets néfastes sur l'activité de production.

Les structures fiscales, dans le cas du Congo-Brazzaville reposent sur les administrations des douanes et impôts. La fiscalité congolaise est de type déclaratif. Elle tire ses ressources de trois principales structures fiscales notamment : les impôts sur les revenus des personnes physiques (IRPP), les impôts sur le bénéfice des sociétés (ISS) et la taxe sur la valeur ajoutée (PNUD, 2012, 182). Ces impôts rapportent à l'Etat près de $80 \%$ des recettes fiscales. Outre ces structures fiscales, on retrouve une multitude d'autres impôts, tels que les impôts sur le revenu, impôts sur la propriété, droits d'enregistrement et du timbre et autres structures fiscales diverses de l'Organisation pour l'Harmonisation en Afrique du Droit des Affaires (OHADA). 
Au regard de ce qui précède, la question est de savoir quels ont été les effets des structures fiscales sur la croissance économique au CongoBrazzaville sur la période de 1980 à 2015 ?

Dans cet article, contrairement aux travaux de Padovano et Galli (2001), Wildmalm (2001), Johansson et al., (2008) et Vaillancourt et Pouya (2012) qui analysent l'impact des structures fiscales sur la croissance, en ignorant les autres catégories des structures fiscales, nous allons mettre l'accent sur les transferts de l'Etat aux entreprises et sur la distinction entre impôts sur les revenus des personnes physiques, impôts sur les sociétés et impôts sur les biens et services. Nous pensons par là mieux comprendre quels impôts affectent positivement ou négativement la croissance économique et donner des pistes quant à ce que devrait être une structure fiscale favorable au développement du Congo-Brazzaville. Pour ce faire, nous mobilisons le modèle économétrique de Dickey et Fuller pour mesurer les effets réels des impôts sur la croissance. Cette étude est à notre connaissance, la première prospection empirique sur l'incidence des structures fiscales sur la croissance économique au Congo-Brazzaville.

Notre article comporte quatre sections. Dans la première, nous présentons la revue de la littérature relative aux liens existants entre les structures fiscales et la croissance économique. Dans la seconde, nous examinons l'évolution des structures fiscales dans un contexte de croissance économique au Congo. Dans la troisième, nous présentons la méthodologie d'analyse adoptée. Dans la quatrième section, nous estimons et interprétons les résultats obtenus. Enfin, nous nous efforcerons d'en dégager les limites de politiques économiques mises en œuvre au Congo et de proposer quelques suggestions.

\section{Revue de la littérature}

La problématique des effets des structures fiscales sur la croissance économique a fait l'objet de nombreuses études théoriques et empiriques.

\section{Revue théorique}

La littérature économique sur les impacts des structures fiscales sur la croissance économique a été marquée par des auteurs tels que Colm et Helzner (1958), Musgrave (1969), Rawls (1971) et Gilbert (1979). Dans leurs travaux sur les structures économiques, Colm et Helzner (1958) montrent que l'évolution des structures fiscales est fonction de trois phases du développement économique : la croissance économique, le décollage et la civilisation de l'abondance. Musgrave (1969) explore les structures fiscales et les leviers fiscaux. Il constate que les structures économiques influencent les structures fiscales au travers de la création de nouvelles bases d'imposition potentielles. Il conclu que les structures fiscales se caractérisent 
par un fort impôt foncier tandis que les impôts sur la consommation doivent se limiter à certains biens de base produits dans des entreprises de grande taille qui fournissent une matière imposable facile à atteindre.

Pour Rawls (1971), l'accent est mis sur les principes d'équité, il estime que l'agent économique, qui doit définir les différents types et niveaux d'impositions, doit se comporter comme s'il se trouvait dans un état originel, qui choisit entre différents états sociaux, en ignorant non seulement sa propre situation, mais aussi les caractéristiques possibles des états sociaux alternatifs.

Gilbert (1979), quant à lui, qualifie les structures fiscales comme étant la répartition du tout ou partie des prélèvements obligatoires, selon un critère de classification déterminée. Autrement dit, les structures fiscales ne sont rien d'autres que des vecteurs dont l'évolution dans le temps ou l'espace peut être observée en les décomposant impôts par impôts ou grâce à l'usage d'indices synthétiques (indice de visibilité, concentration apparente...). Selon lui, les structures fiscales ont pour objectif de comprendre la portée des lois d'évolution de la composition du prélèvement fiscal et la signification qu'y prend la dynamique des différents impôts et cotisations sociales. Leur évolution passe par la préexistence de lois d'évolution des dépenses publiques dont l'aspect fiscal constitue l'envers du décor, par le développement d'une théorie des opportunités d'imposition dont bénéficient les autorités. Il confirme que les structures fiscales s'appliquent aux qualités apparentes des impôts (assiettes, modes d'imposition...) ainsi qu'à ses effets (psychologiques et politiques, fraude...). Il existe plusieurs types de structures fiscales (impôts sur le revenu des personnes physiques, impôts sur les sociétés, impôts sur les revenus de capitaux mobiliers...) et de critères pour les classer (assiette, agent collecteur, administratif...). Toutefois, ces critères concernent aussi le degré de libéralisme, le degré de socialisme net, la compétitivité internationale, la neutralité, la justice et la productivité de l'impôt (Bracewell-Milness, 1976).

Romer (1986), Lucas (1988) et Aghion et Howitt (1988) vont plus loin en soulignant que la fiscalité est un outil de la croissance économique : une meilleure politique fiscale encourage les entreprises à investir dans le capital physique et dans l'innovation. Cela favorise la création de richesses, des emplois et permet d'élargir l'assiette fiscale. Slemrod (1990) constate que les structures fiscales ne peuvent être mises en place sans dispositif coercitif visant à assurer la collecte effective de l'impôt.

\section{Revue empirique}

De nombreux travaux empiriques ont été réalisés sur les effets des structures fiscales sur la croissance économique dans un certain nombre de pays développés et en développement. Milesi-Feretti et Roubini (1998) par 
exemple, modélisent dans les pays riches, une économie à trois secteurs, notamment le secteur des biens finaux, le secteur du capital humain et des biens non marchands pour analyser l'incidence de l'imposition du revenu et de la consommation sur la croissance économique. Ces auteurs confirment que la taxe sur la consommation réduit la croissance, avec pour conséquence une distorsion entre le temps passé en loisir et le temps passé aux activités productives. Pour ces auteurs, l'impôt sur le revenu est à l'origine de cette distorsion. Dans le cas de la taxe sur la consommation, ils concluent que l'imposition du revenu entraîne d'autres distorsions qui diminuent l'accumulation du capital et donc la croissance économique.

Padovano et Galli (2001) dans une étude sur les effets de l'imposition du revenu personnel sur la croissance économique pour un panel de 23 pays de l'OCDE sur la période de 1951 à 1990, trouvent une relation négative et significative entre le taux marginal de taxation et le taux de croissance moyen du produit intérieur brut (PIB), pour un niveau donné de croissance démographique, de capital physique et de capital humain. Ils confirment aussi l'effet négatif de la progressivité de la structure fiscale sur l'allocation des ressources.

Widmalm (2001) examine les effets de l'impôt sur le revenu personnel, l'impôt sur le revenu corporatif, les taxes sur les biens et services et l'impôt foncier sur la croissance économique. Pour cet auteur, la progressivité d'une taxe à des effets négatifs sur la croissance économique.

Young et Gordon (2004) étudient l'impact de l'imposition du revenu personnel, notamment de l'imposition du revenu corporatif sur la croissance économique. En analysant dans une première phase, les effets des taux statutaires d'imposition du revenu personnel et du revenu corporatif sur la croissance, ils constatent qu'une augmentation de $10 \%$ du taux d'imposition du revenu corporatif induit une baisse de $0,82 \%$ du taux de croissance. En estimant la part des recettes fiscales provenant de l'impôt sur le revenu personnel en fonction du taux d'imposition du revenu corporatif, ces auteurs obtiennent une corrélation positive entre ces variables, résultats qu'ils justifient par l'entreprenariat, c'est-à-dire le nombre d'individus déclarant un revenu corporatif se réduit au fur et à mesure que le taux d'imposition du revenu corporatif augmente. De plus, ces individus trouvent plus avantageux de déclarer un revenu personnel plutôt qu'un revenu corporatif, ce qui réduirait davantage leur fardeau fiscal (Vaillancourt et Pouya, 2012).

Keller (2004), Griffith et al., (2004) et Bloom et al., (2007) s'interrogent sur les effets des taux de l'impôt sur les sociétés et l'investissement direct étranger dans les pays développés. Pour eux, une augmentation des taux de l'impôt sur les sociétés, décourage l'investissement direct étranger et, donc, la présence d'entreprises multinationales étrangères, 
ce qui se traduit par des effets négatifs des taux de l'impôt sur les sociétés sur la productivité.

Johansson et $a l .$, (2008) ont étudié la relation entre le système fiscal et la croissance à long terme. Pour ce faire, ils ont utilisé un panel de 21 pays de l'OCDE sur une période de 34 années pour analyser l'impact de la taxation sur la croissance à court et moyen termes. Ils trouvent que la taxation de la consommation et l'imposition des biens immobiliers sont les moins nuisibles à la croissance économique, alors que les impôts sur le revenu corporatif et les impôts sur le revenu personnel sont les plus nuisibles. Ils constatent également qu'une augmentation du taux d'imposition du revenu corporatif diminue la productivité totale des facteurs et celle de l'investissement au niveau des firmes. Et, une augmentation du taux marginal d'imposition du revenu personnel a des effets néfastes importants sur la productivité totale des facteurs pour les industries où l'entrée de nouvelles firmes est plus fréquente.

Les travaux de l'OCDE (2009) analysent l'impact des impôts sur les bénéfices des sociétés, des investissements nationaux, investissements étrangers et des incitations des entreprises à investir dans des activités novatrices sur la production. Ces travaux montrent une incidence négative entre ces variables et la production. Ils confirment également les effets négatifs des impôts sur le revenu du travail, des cotisations de sécurité sociale sur les incitations des travailleurs à fournir de la main-d'œuvre.

Ngoc et Arseneau (2012) sur la base d'un échantillon des pays de l'OCDE et sur une période de 36 années, étudient l'incidence de l'imposition $\mathrm{du}$ revenu personnel, le revenu corporatif, la masse salariale et la consommation sur la croissance du PIB par habitant. Ces auteurs utilisent dans un premier temps, le ratio des trois premiers types de taxes relatives à la consommation qu'ils transforment sous la forme logarithmique. Dans un deuxième temps, ils considèrent la moyenne de cinq années de toutes les variables incluses dans le modèle pour éliminer l'auto-corrélation. Ils obtiennent une relation négative entre le ratio des recettes fiscales de l'impôt sur le revenu sur les recettes de la taxe à la consommation et la croissance, mais aussi un impact positif entre la taxation de la consommation et la croissance économique.

Vaillancourt et Pouya (2012) mesurent l'impact de la taxation sur le taux de croissance du PIB dans les provinces canadiennes pour la période 1981 à 2010. Ils utilisent les recettes fiscales, la taxe à la consommation, l'impôt sur le revenu corporatif et l'impôt sur le revenu du personnel. Ils trouvent que l'impôt corporatif et la taxe à la consommation exercent des effets négatifs sur le taux de croissance du PIB par rapport à l'impôt sur le revenu personnel. De plus, le niveau initial du PIB impacte significativement 
le taux de croissance du PIB, recommandant que l'ouverture au commerce apparaisse comme un facteur favorable au progrès économique.

Plusieurs autres travaux, notamment Koester et Kormendi (1989), Tanzi et Zee (1997), Mendoza, Milesi-Ferretti et Asea (1997), Brun et al., (1998), Kneller et al., (1999), Adams et al., (2005), Felix (2006), Hasset et Mathur (2006), Arulampalan et al., (2009) et Fayçal et Saloua (2016) analysent les incidences des structures fiscales sur la croissance économique dans les pays développés. Koester et Kormendi (1989) explorent sur 63 pays pour la période de 1970 à 1979, les effets des taux d'imposition moyens sur la croissance économique. Ils constatent que les taux d'imposition moyens n'affectent pas la croissance économique. Tanzi et Zee (1997) dans leurs travaux consacrés aux politiques budgétaires et la croissance à long terme, mettent en évidence un effet négatif des impôts sur les revenus sur l'investissement et la croissance. Mendoza, Milesi-Ferretti et Asea (1997), suggèrent que l'impact de la politique fiscale sur la croissance économique est négligeable. Brun et al., (1998) dans un échantillon de 78 pays développés, trouvent deux effets négatifs des structures fiscales sur la croissance : l'effet de la fiscalité assise sur le commerce extérieur sur la croissance et l'effet du taux de prélèvement global sur la croissance. Kneller et al., (1999) dans une étude sur les pays de l'OCDE, montrent que les impôts sur les revenus, les bénéfices et les cotisations sociales réduisent la croissance. La même étude confirme que les impôts non distortionnaires, tels que la taxation des biens et services n'ont pas d'impact significatif sur la croissance. En revanche, Adams et al., (2005) soulignent que le niveau global d'imposition ou le dosage impôts directs/indirects n'a pas d'effet sur la croissance.

Pour sa part, Felix (2006) montre qu'une augmentation de $10 \%$ de l'impôt sur les sociétés diminue le salaire brut annuel de 7\%. Son estimation pour les Etats-Unis conduit à une charge sur le travail quatre fois plus importante que le montant de l'impôt sur les sociétés. Hasset et Mathur (2006) vont aussi dans le même sens. Pour ces auteurs, une hausse de $1 \% \mathrm{du}$ taux de l'impôt sur les sociétés s'accompagne d'une baisse de $1 \%$ du taux de salaire.

Si Arulampalan et al., (2009) estiment qu'un 1\$ d'impôt sur les bénéfices réduit les salaires de 92 centimes à long terme, par contre, Fayçal et Saloua (2016) dans leurs travaux sur l'impact des recettes fiscales sur la croissance économique dans les grands pays et les paradis fiscaux, attestent que l'impôt est un outil important pour faire face à la récession actuelle et au ralentissement économique. Par ailleurs, l'impôt contribue à la croissance à long terme dans les deux groupes de pays.

Dans les pays en développement, très peu d'études, à notre connaissance, semblent avoir analysé l'incidence des structures fiscales sur 
la croissance économique, exceptées celles de Yaya Keho (2010) et Ebeke et Ehrhart (2010). Yaya Keho (2010) s'est intéressé au problème des prélèvements fiscaux sur l'activité économique en Côte d'Ivoire. En utilisant les tests de cointégration et de causalité sur des données annuelles couvrant la période de 1960 à 2006, l'auteur obtient à long terme, l'existence de relations entre la consommation, l'investissement et le PIB. À court terme, il trouve une relation négative entre les taxes sur les biens et services, les taxes sur le commerce et la croissance du PIB par tête. Pour l'auteur, la fiscalité ne freine pas la croissance à long terme et les recettes fiscales sont positivement corrélées au PIB et à ses composantes. En revanche, Ebeke et Ehrhart (2010) concluent que d'autres effets des structures fiscales sur la croissance proviennent de la forte instabilité des recettes fiscales dans de nombreux pays en développement.

Cette revue de la littérature suggère que dans les pays émergents et dans les pays peu développés, les travaux théoriques mettent l'accent sur l'équité, pour justifier les effets des structures fiscales sur la croissance. Alors que les travaux empiriques insistent sur les variables, telles que les impôts à la consommation, l'investissement, les taxes sur le commerce, les taxes sur les biens et services et les recettes fiscales pour expliquer l'impact des structures fiscales sur la croissance économique. Il parait donc pertinent d'analyser l'évolution des structures fiscales dans un contexte de croissance économique dans un pays en développement en l'occurrence, le CongoBrazzaville.

\section{Evolution des structures fiscales et dynamique de croissance au Congo- Brazzaville}

Dès les années 1960, la dynamique de croissance économique et celle des structures fiscales au Congo a été irrégulière. Ce pays a enregistré des faibles taux de croissance jusqu'à la fin des années 1990, avant de connaitre dans la première décennie des années 2000, un regain de croissance forte et continue (CNUCED, 2014, 2). Le taux de croissance moyen et le taux moyen des impôts sur les revenus du Congo ont été respectivement, de l'ordre de $4,19 \%^{2}$ et $33,57 \%^{3}$ sur la période de 1980 à 2015 , correspondant ainsi, aux taux des pays à revenu intermédiaire de la tranche inférieure.

Dans ce travail, nous analyserons l'évolution des structures fiscales à partir des impôts sur les revenus. Ces impôts sont regardés comme prioritaires dans tous les débats sur les effets de la politique fiscale (OCDE, 2009). Pour mieux appréhender leur évolution, il faudrait distinguer deux grandes périodes : 1980 à 2005 et 2006 à 2015.

2 Taux calculé par l'auteur, à partir des données de la Banque mondiale.

3 Taux calculé par l'auteur, à partir des données de la BEAC. 
Graphique 1 : Evolution des impôts sur les revenus

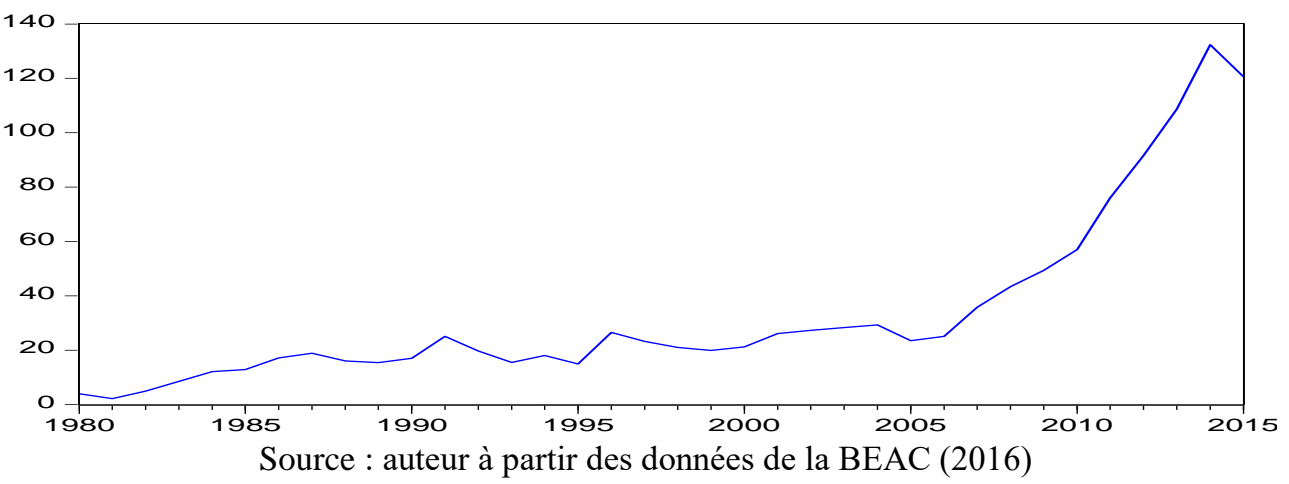

La première période va de 1980 à 2005 , et se caractérise par une évolution assez irrégulière des impôts sur les revenus dans le temps. Cette irrégularité peut s'expliquer par une instabilité des impôts, ce qui rend, par ailleurs, difficile leur prévision. La tendance à la baisse de ces impôts sur cette période montre que les impôts sur les revenus croissent moins vite que le PIB. Ce qui laisse supposer que ces impôts sont en deçà de ce qu'ils auraient été si le taux de pression fiscale avait été maintenu constant à leurs niveaux.

Plusieurs facteurs ont été mis en avant pour justifier ces variations. En premier lieu, la structure trop centralisée de l'administration fiscale congolaise, au cours de la première moitié des années 1980. En second lieu, l'irrégularité des impôts sur les revenus tient au poids excessif de la fiscalité et de la parafiscalité. En effet, au cours de cette période, le taux de pression fiscale du pays a été en moyenne au-dessus de $19 \%$ (PND, 2012 - 2016). Cette situation a entraîné chez le contribuable un manque de civisme fiscal, car les contribuables soumises à l'imposition ont été incités à déclarer moins d'impôts. Troisièmement, cette irrégularité des impôts a occasionné le développement des pratiques de fraude et de corruption de certains agents administratifs qui se sont traduits par une parafiscalité illégale et onéreuse. Selon une étude de l'Union Européenne, les entreprises congolaises paieraient 2 à 3 fois plus d'impôts que dans les autres pays africains.

L'irrégularité des impôts au cours de cette période pourrait également s'expliquer par l'effondrement des cours mondiaux des matières premières en 1985, notamment du pétrole, entraînant ainsi la baisse des recettes publiques de l'Etat, des investissements, des transferts de l'Etat aux entreprises, dépenses publiques, recettes budgétaires d'exportation et d'accumulation des déficits budgétaires et extérieurs entre 1985 et 1989 (PNUD, 2012, 36). 
La deuxième période va de 2006 à 2015, elle est marquée jusqu'en 2013 par une forte tendance à l'augmentation des impôts sur les revenus. Entre 2008 et 2011, les recettes de l'Etat ont augmenté de $10 \%$ à $13 \%$, de même, le taux moyen des impôts sur le revenu en ratio du PIB est passé de $23 \%$ à $25 \%$ (PND 2012-2016). L'accroissement des impôts résulterait de nombreuses réformes fiscales mises en place par le gouvernement pour répondre aux besoins d'amélioration du rendement fiscal. Parmi ces réformes, la plus importante est celle de 2011-2013 relative à la réforme de l'IRPP, notamment, l'allégement de la pression fiscale. Comme le suggère Laffer (2004), une amélioration de la pression fiscale entraîne une absence de la fraude et de l'évasion fiscale, donc un changement de comportement fiscal de la part des agents économiques et des fonctionnaires du fisc. Mais aussi, une sincérité dans les déclarations des contribuables, une transparence dans les transactions des entreprises et une bonne gouvernance dans la gestion des affaires imposables (Ngakosso, 2016). Aussi, cette reforme s'est traduite par la réduction du taux de chaque tranche d'imposition de 5 points à l'exception de la première tranche qui est restée à $1 \%$. Ce taux est passé de $26,5 \%$ en 2011 à 23,5\% du PIB en 2015, profitant aux ménages à revenu élevé et célibataires avec un niveau intermédiaire de revenu (FMI, 2014).

Ces réformes vont porter également sur l'incitation à la distribution des dividendes, l'attraction des investissements étrangers directs, les investissements internationaux de portefeuille, la réduction du secteur informel en vue de lutter contre la concurrence déloyale et le faible niveau de la bancarisation de l'économie. En effet, la bancarisation de l'économie permet de lutter contre les transactions qui échappent au contrôle du fisc. À cela s'ajoutent l'élargissement de l'assiette fiscale, la promotion du civisme fiscal par l'assistance et la sensibilisation des opérateurs privés, l'abattement fiscal sur le revenu salarial et le régime applicable aux familles et la modernisation du système fiscal par la simplification des procédures en vue de réduire la perte de temps aux contribuables. Tous ces éléments ont aussi contribué à la hausse des impôts sur le revenu durant cette période.

À partir de 2014, d'autres réformes fiscales modernes ont vu le jour. Ces réformes avaient pour objectif de renforcer les précédentes. Comme le souligne la direction générale des impôts et des domaines (DGID, 2016), le système global d'imposition des revenus du Congo, ayant montré ses insuffisances au cours de la période de 2011-2013 en raison de la nonsouscription des déclarations par les détenteurs des différentes catégories de revenu, les pouvoirs publics ont mis en place de nouvelles réformes en 2014. Ainsi, ces réformes ont porté notamment sur l'IRPP, la réduction de 33 à $30 \%$ du taux de l'impôt sur les sociétés, la réforme de la patente, la réduction du nombre d'échéances déclaratives et de paiements des impôts, l'institution du régime fiscal des zones économiques spéciales et industrielles, 
l'institution de la taxe d'occupation des locaux et du régime fiscal des zones franches de santé. Malgré ces réformes louables, le Congo a connu une chute des impôts sur le revenu de 2014 à 2015.

Il ressort de l'évolution des impôts sur le revenu au Congo, que ceuxci ont connu une forte tendance à la hausse. Pour mieux cerner cette problématique, il convient de mener une étude empirique dans le contexte congolais qui nous permettra de confronter les approches théoriques à la réalité, afin de faire ressortir quelques éléments de politique économique permettant de bien appréhender les problèmes des effets des structures fiscales sur la croissance économique au Congo-Brazzaville.

\section{Méthodologie \\ Modèle théorique}

Bon nombre des modèles économétriques ont été utilisés pour étudier l'impact des structures fiscales sur la croissance économique, notamment Mankiw et al., (1992), Islam (1995), Caselli et al., (1996), Barro (1996) et Widmalm (2001). Ces auteurs ont utilisé pour la plupart des modèles en panel. Dans cet article nous allons nous appuyer sur une fonction de type Cobb-Douglas pour établir les liens existants entre la structure des impôts et la croissance économique. Cette modélisation nous semble la mieux appropriée pour rendre compte des effets des structures fiscales dans le cadre d'une économie extravertie et dépendante des ressources pétrolières :

$$
Y=A K^{\alpha} . L^{\beta} ; \quad \alpha, \beta \geq 0 .
$$

Avec $Y$ : niveau de production, $A:$ progrès technique, $K$ : capital physique, $L:$ travail.

Une amélioration de la productivité peut être le résultat d'une réelle politique des structures fiscales (STF) et d'une réorganisation de ces structures reposant sur un transfert de la charge fiscale de l'impôt sur le revenu vers l'impôt sur la consommation, ce qui rendrait le système fiscal plus efficace et favorable à la croissance économique (Vartia, 2008; Johansson, 2011). Au Congo, cette réorganisation passe par une meilleure gestion des recettes de l'Etat (RTE) et des transferts d'une partie de ces recettes aux entreprises (TRE).

Nous posons donc: $A=S T F+R T E+T R E$, avec $S T F=I R P+I S S+I B S$

Soit $A=I R P+I S S+I B S+R T E+T R E$.

Avec IRP : impôts sur les revenus des personnes physiques; ISS : impôts sur les sociétés ; IBS : impôts sur les biens et services.

Nous pouvons donc écrire l'équation (1) sous la forme suivante :

$Y=(I R P+I S S+I B S+R T E+T R E) K^{\alpha} \cdot L^{\beta}$

(2) 
À l'aide d'une transformation mathématique, nous pouvons écrire ce modèle multiplicatif sous forme linéaire :

$\ln Y=\ln I R P+\ln I S S+\ln I B S+\ln R T E+\ln T R E+\alpha \ln K+\beta \ln L+\varepsilon$

(3)

Avec $K$ : formation brute de capital fixe $(F B C)$ et $L$ : population active (POA).

\section{Modèle empirique et ses variables}

À partir de la relation (3), nous proposons le modèle linéaire empirique suivant :

$$
\begin{aligned}
& \begin{array}{l}
\ln P I B R_{t} \\
+
\end{array}=\alpha_{0}+\alpha_{1} \ln (I R P / P I B)_{t}+\alpha_{2} \ln (I S S / P I B)_{t}+\alpha_{3} \ln (I B S / P I B)_{t}+\alpha_{4} \ln (R T E / P I B)_{t} \\
& \quad \alpha_{5} \ln (T R E / P I B)_{t}+\alpha_{6} \ln (F B C / P I B)_{t}+\alpha_{7} \ln (P O A)_{t+} \varepsilon_{t}
\end{aligned}
$$

Où $\alpha_{0}$ est la constante, $\alpha_{1} \ldots \alpha_{7}$ les paramètres à estimer, $\varepsilon_{t}$ le terme d'erreur, $\varepsilon_{t} \rightarrow N\left(0, \sigma^{2}\right)$.

La variable expliquée est le produit intérieur brut réel (PIBR), les variables explicatives sont, les impôts sur les revenus des personnes physiques rapportés au PIB (IRP/PIB). Ils désignent les impôts prélevés sur le revenu net et les plus-values des personnes physiques. Les impôts sur les sociétés rapportés au PIB (ISS/PIB) qui, sont des impôts sur le revenu des entreprises. Les impôts sur les biens et services rapportés au PIB (IBS/PIB), qui comprennent l'ensemble des impôts prélevés sur la production, l'extraction, la vente, le transfert, la livraison de biens, la prestation de services, l'utilisation de biens et l'autorisation d'utiliser des biens ou d'exercer des activités. Les recettes fiscales totales en pourcentage du PIB (RTE/PIB) indiquent la part de la production d'un pays prélevée par l'État sous forme d'impôts. Les transferts de l'Etat aux entreprises (TRE/PIB) indiquent les ressources que l'Etat transfère aux entreprises. La formation brute de capital fixe (FBC/PIB) est l'investissement des différents agents économiques résidents en capital fixe. Quant à la population active (POA), elle représente la quantité de travail fournie dans une économie.

Les données retenues sont issues de la Banque des Etats de l'Afrique Centrale (BEAC) pour les impôts sur les revenus des personnes physiques, impôts sur les sociétés et impôts sur les biens et services. Celles relatives aux recettes de l'Etat, transferts de l'Etat et au produit intérieur brut réel proviennent du Programme des Nations Unies pour le Développement (PNUD). Les données sur la formation brute de capital fixe et la population active sont tirées de la Banque Mondiale. Les données couvrent la période allant de 1980 à 2015. Nous justifions ce choix par l'indisponibilité des données statistiques. 


\section{Estimation du modèle et interprétation des résultats Estimation du modèle}

L'estimation du modèle requiert que nous étudions d'abord les tests de racine unitaire et celui de la cointégration.

\section{Tests de racine unitaire}

Les tests de racine unitaire permettent de détecter la présence de racine unitaire dans une série. Dans ce travail, nous avons choisi le test de Dickey et Fuller Augmenté (ADF) et le test de Phillips et Perron (PP). Les résultats des tests sont présentés dans le tableau ci-dessous.

Tableau 1 : Test de stationnarité ADF et PP

\begin{tabular}{|c|c|c|c|c|c|c|}
\hline \multirow{2}{*}{ Variables } & \multicolumn{2}{|c|}{ Niveau } & \multicolumn{2}{c|}{ Différence première } & \multicolumn{2}{c|}{ Décisions } \\
\cline { 2 - 7 } & ADF & PP & ADF & PP & ADF & PP \\
\hline LPIB & -0.677377 & -0.664454 & -5.957248 & -6.426470 & $\mathrm{I}(1)$ & $\mathrm{I}(1)$ \\
\hline LIRP_PIB & -1.497297 & -1.456671 & -5.966489 & -6.011705 & $\mathrm{I}(1)$ & $\mathrm{I}(1)$ \\
\hline LISS_PIB & -2.293297 & -2.135121 & -8.204840 & -9.287262 & $\mathrm{I}(1)$ & $\mathrm{I}(1)$ \\
\hline LIBS_PIB & -0.120986 & -1.164917 & -7.985230 & -10.05936 & $\mathrm{I}(1)$ & $\mathrm{I}(1)$ \\
\hline LRTE_PIB & -1.170900 & -1.946792 & -8.203988 & -9.031878 & $\mathrm{I}(1)$ & $\mathrm{I}(1)$ \\
\hline LTRE_PIB & -0.889127 & -0.973933 & -5.891870 & -5.907810 & $\mathrm{I}(1)$ & $\mathrm{I}(1)$ \\
\hline LFBC_PIB & -2.439054 & -2.467907 & -6.336199 & -6.345448 & $\mathrm{I}(1)$ & $\mathrm{I}(1)$ \\
\hline LPOA & -0.025765 & 0.556458 & -2.844029 & -3.371561 & $\mathrm{I}(1)$ & Non \\
\hline
\end{tabular}

Les résultats obtenus indiquent que toutes les variables de l'étude sont stationnaires en différence première, sauf la $P O A$. Le degré de significativité est de 5\%. Puisque la variable $P O A$ n'est pas stationnaire en différence première, on la retire du modèle (4). On obtient alors, la spécification suivante du modèle :

$$
\begin{aligned}
& \ln P I B R_{t}=\alpha_{0}+\alpha_{1} \ln (I R P / P I B)_{t}+\alpha_{2} \ln (I S S / P I B)_{t}+\alpha_{3} \ln (I B S / P I B)_{t}+\alpha_{4} \ln (R T \\
& E / P I B)_{t}+\quad \alpha_{5} \ln (T R E / P I B)_{t}+\alpha_{6} \ln (F B C / P I B)_{t+} \varepsilon_{t}
\end{aligned}
$$

À partir de ce modèle, on peut procéder au test de cointégration pour vérifier si ces variables sont cointégrées.

\section{Test de Cointégration et test de valeur propre maximale}

Plusieurs tests (test d'Engle-Granger, test de Johansen...) sont utilisés pour déterminer s'il y a ou non de la cointégration entre les variables. Dans cette étude, nous retenons le test de Johansen et celui de la valeur propre maximale qui indiquent la quantité de relation de cointégration. Les résultats de ces tests sont résumés dans les tableaux ci-après. 
Tableau 2: Résultats du test de cointégration

\begin{tabular}{|c|c|c|c|c|}
\hline $\begin{array}{c}\text { Hypothesized } \\
\text { No. of CE(s) }\end{array}$ & Eigenvalue & $\begin{array}{c}\text { Trace } \\
\text { Statistic }\end{array}$ & $\begin{array}{c}0.05 \\
\text { Critical Value }\end{array}$ & Prob** \\
\hline None* & 0.779582 & 150.2345 & 125.6154 & 0.0007 \\
\hline At most1* & 0.775309 & 98.81868 & 95.75366 & 0.0302 \\
\hline At most2* & 0.465806 & 48.05570 & 69.81889 & 0.7196 \\
\hline At most3* & 0.260008 & 26.73786 & 47.85613 & 0.8640 \\
\hline At most4 & 0.233393 & 16.49992 & 29.79707 & 0.6767 \\
\hline At most5 & 0.139793 & 7.463360 & 15.49471 & 0.5244 \\
\hline At most 6 & 0.066607 & 2.343569 & 3.841466 & 0.1258 \\
\hline
\end{tabular}

Trace test indicates 2 cointegrating eqn(s) at the 0.05 level

* denotes rejection of the hypothesis at the 0.05 level

**MacKinnon-Haug-Michelis (1999) p-values.

Tableau 3 : Résultats du test de valeur propre maximale

\begin{tabular}{|c|c|c|c|c|}
\hline $\begin{array}{c}\text { Hypothesized } \\
\text { No. of CE(s) }\end{array}$ & Eigenvalue & $\begin{array}{c}\text { Max-Eigen } \\
\text { Statistic }\end{array}$ & $\begin{array}{c}0.05 \\
\text { Critical Value }\end{array}$ & Prob $^{* *}$ \\
\hline None & 0.779582 & 51.41582 & 46.23142 & 0.0128 \\
\hline At most 1 $*$ & 0.775309 & 50.76298 & 40.07757 & 0.0022 \\
\hline At most 2 & 0.465806 & 21.31784 & 33.87687 & 0.6602 \\
\hline At most 3 & 0.260008 & 10.23794 & 27.58434 & 0.9813 \\
\hline At most 4 & 0.233393 & 9.036560 & 21.13162 & 0.8295 \\
\hline At most 5 & 0.139793 & 5.119790 & 14.26460 & 0.7266 \\
\hline At most 6 & 0.066607 & 2.343569 & 3.841466 & 0.1258 \\
\hline
\end{tabular}

Max-eigenvalue test indicates 2 cointegrating eqn(s) at the 0.05 level

* denotes rejection of the hypothesis at the 0.05 level

**MacKinnon-Haug-Michelis (1999) p-values.

Les résultats du test de Johansen et celui de la valeur propre maximale montrent que les variables retenues sont cointégrées au seuil de $5 \%$. L'hypothèse nulle d'absence de cointégration, est rejetée du fait que le test de la trace et le test de Max-eigenvalue indiquent chacun deux équations de cointégration. Il est donc possible d'identifier (7-2) $=5$ impulsions persistantes et les autres constituent une source d'aléas qui n'exercent qu'un effet transitoire. Ces résultats suggèrent l'existence d'au moins une relation de long terme entre les variables. Les résultats de l'estimation des modèles de long terme et de court terme obtenus à partir du modèle économétrique sont présentés dans les tableaux 4 et 5 . Les deux modèles ont subi les tests d'autocorrélation et d'hétéroscédasticité. 
Tableau 4 : Résultats de l'estimation du modèle de long terme

\begin{tabular}{|cclcl|}
\hline Variable & Coefficient & Std. Error & t-Statistic & Prob. \\
\hline C & 7.306448 & 0.863796 & 8.458538 & 0.0000 \\
LIRP_PIB & -0.318674 & 0.104075 & -3.061973 & 0.0047 \\
LISS_PIB & 0.008367 & 0.146051 & 0.057287 & 0.9547 \\
LIBS_PIB & 0.237126 & 0.110588 & 2.144225 & 0.0405 \\
LRTE_PIB & 0.845919 & 0.149347 & 5.664122 & 0.0000 \\
LTRE_PIB & -0.431875 & 0.048097 & -8.979218 & 0.0000 \\
LFBC_PIB & -0.340655 & 0.102935 & -3.309408 & 0.0025 \\
\hline \hline R-squared & 0.969951 & Mean dependent var & 7.425645 \\
Adjusted R-squared & 0.963734 & S.D. dependent var & 0.897463 \\
S.E. of regression & 0.170908 & Akaike info criterion & -0.522712 \\
Sum squared resid & 0.847081 & Schwarz criterion & -0.214805 \\
Log likelihood & 16.40882 & Hannan-Quinn criter. & -0.415244 \\
F-statistic & 156.0173 & Durbin-Watson stat & 1.163123 \\
Prob(F-statistic) & 0.000000 & & & \\
\hline
\end{tabular}

Tableau 5 : Résultats de l'estimation du modèle de court terme

\begin{tabular}{|ccccc|}
\hline Variable & Coefficient & Std. Error & t-Statistic & Prob. \\
\hline C & 0.052185 & 0.017525 & 2.977691 & 0.0061 \\
D(LIRP_PIB) & -0.091940 & 0.088623 & -1.037423 & 0.3087 \\
D(LISS_PIB) & -0.140419 & 0.070115 & -2.002705 & 0.0553 \\
D(LIBS_PIB) & -0.068469 & 0.068415 & -1.000785 & 0.3258 \\
D(LRTE_PIB) & 0.429144 & 0.095424 & 4.497250 & 0.0001 \\
D(LTRE_PIB) & -0.258386 & 0.054489 & -4.741959 & 0.0001 \\
D(LFBC_PIB) & -0.114939 & 0.077107 & -1.490641 & 0.1476 \\
RESID01(-1) & -0.426994 & 0.116942 & -3.651320 & 0.0011 \\
\hline \hline R-squared & 0.774537 & Mean dependent var & 0.086196 \\
Adjusted R-squared & 0.716083 & S.D. dependent var & 0.172912 \\
S.E. of regression & 0.092134 & Akaike info criterion & -1.733511 \\
Sum squared resid & 0.229195 & Schwarz criterion & -1.378003 \\
Log likelihood & 38.33645 & Hannan-Quinn criter. & -1.610790 \\
F-statistic & 13.25049 & Durbin-Watson stat & 2.000423 \\
Prob(F-statistic) & 0.000000 & \multicolumn{3}{c}{} \\
\hline
\end{tabular}

Interprétation et discussion des résultats

Interprétation des résultats

Il ressort des résultats sur les effets des structures fiscales sur la croissance à long et court termes, que les tests d'autocorrélation et d'hétéroscédasticité effectués, suggèrent fortement que les deux modèles sont homoscédastiques et qu'il n'y a pas d'auto-corrélation dans les résidus du modèle (tableaux 6 et 7 en annexe).

Dans ces résultats, les statistiques de Fischer (F) sont importantes ainsi que les probabilités qui y sont associées sont nulles. Aussi, le pouvoir explicatif de ces variables est estimé à $97 \%$ pour le modèle de long terme et à $77 \%$ pour le modèle de court terme. Ces résultats montrent que les 
variables explicatives contribuent à l'explication de la variabilité totale. Ils révèlent que, les coefficients de détermination sont suffisamment élevés. En conséquence, les modèles de long terme et de court terme sont globalement satisfaisants.

Dans le modèle de court terme, le coefficient affecté à la variable mesurant la vitesse d'ajustement (-0.426994) est statistiquement négatif et significatif au seuil de $5 \%$. Ce résultat confirme l'existence d'une relation stable à long terme entre les structures fiscales et les déterminants de la croissance économique.

Ainsi, à long terme, l'analyse des résultats indique qu'au Congo, cinq variables affectent la croissance économique. Ces variables sont les impôts sur les revenus des personnes physiques, les impôts sur les biens et services, les recettes totales de 1'Etat, les transferts de l'Etat et la formation brute de capital fixe. Les résultats empiriques de notre étude nous enseignent que lorsque les impôts sur les revenus des personnes physiques augmentent de 1 $\%$ sur la période étudiée, toute chose égale par ailleurs, cela se traduit par une diminution de $0,31 \%$ de croissance. En ce qui concerne les impôts sur les biens et services, nous constatons que ceux-ci exercent une influence positive sur la croissance. Une hausse de $1 \%$ de ces impôts, entraîne une hausse de 0,23\% de croissance. Pour les recettes de l'Etat, nous observons qu'une augmentation de $1 \%$ de ces recettes implique une augmentation de $0,84 \%$ de croissance. Par contre, pour les transferts de l'Etat, une augmentation de $1 \%$ de ces transferts induit une diminution de la croissance économique de l'ordre de $0,43 \%$. Quant à la formation brute de capital fixe, nous observons qu'une augmentation de $1 \%$ de ce capital physique se traduit par une diminution de la croissance de l'ordre de $0,34 \%$.

À court terme, l'analyse des coefficients montre que trois variables exercent des effets significatifs au seuil de $5 \%$ sur la croissance. Les impôts sur les sociétés l'affectent négativement. Une hausse de ces impôts de $1 \%$, toute chose égale par ailleurs, induit une baisse de la croissance de 0,14\%. Les recettes totales de l'Etat impactent positivement la croissance. Une hausse de ces recettes de $1 \%$, entraîne une hausse de la croissance de $0,42 \%$. En revanche, les transferts de l'Etat aux entreprises ont des effets négatifs sur la croissance. Une augmentation de $1 \%$ de ces transferts, se traduit par une baisse de croissance de l'ordre de $0,25 \%$.

\section{Discussion des résultats d'étude}

Sur la période étudiée, notre étude montre que les recettes de l'Etat exercent une influence positive sur la croissance économique congolaise. $\mathrm{Ce}$ résultat a été mis en évidence par Widmalm (2001) selon lequel la part des dépenses gouvernementales provenant notamment des recettes fiscales contribue à améliorer la productivité. Mieux encore, il vient conforter les 
positions de Keen et al., (2010 a) et Yaya Keho (2010) qui confirment qu'en Afrique subsaharienne, les résultats des recettes fiscales sont meilleurs que dans les pays riches et par conséquent, amélioreraient leur croissance. Au Congo-Brazzaville, ces résultats suggèrent que l'accroissement des recettes budgétaires est supérieur aux distorsions liées aux instruments de collectes de ces revenus. Cela signifie que les recettes de l'Etat sont dépendantes et liées aux ressources pétrolières, ce qui montre que le pétrole dispose d'un impact positif dans la croissance de l'économie congolaise.

A long terme, cette étude s'oppose aux résultats de Fayçal et Saloua (2016) et ceux de Lakhdar et al., (2017). Mais confirme celles de Tanzi et Zee (1997), Kneller et al., (1999), Widmalm (2001) et Johansson et al., (2008), selon lesquelles la progressivité de l'impôt sur les revenus des personnes physiques crée une distorsion dans le choix travail-loisir, ce qui se traduit par une réduction de la croissance. Dans le cas du Congo, cela signifie qu'il vaudrait mieux limiter le recours à ce levier si on veut favoriser la croissance (Ades et al., 2015).

A court terme, cette étude indique que les impôts sur les sociétés ont un impact négatif sur la croissance. L'effet négatif des impôts sur les sociétés sur la croissance a déjà été mis en évidence par plusieurs études empiriques, notamment celles de Levine et Renelt (1992), Felix (2006), Bloom et al., (2007), Johansson et al., (2008), FMI (2010), Romer (2010) et Gemmel et al., (2011) qui soutiennent qu'une augmentation des taux de l'impôt sur les sociétés, décourage la productivité. Dans le cas du Congo, ce résultat suggère que les impôts sur les sociétés ne contribuent pas au développement du pays : il ressort des estimations effectuées, à long terme, que les impôts sur les sociétés n'ont pas d'influence sur la croissance économique.

Cette étude montre également que contrairement à ce que soutiennent Keller (2004), Griffith et al., (2004), Criscuolo (2006), Felix (2006), Hasset et Mathur (2006) et Bloom et al., (2007), une augmentation des taux de l'impôt sur les biens et services, ne décourage pas la productivité au Congo ou que du moins, ils contribuent à la croissance économique.

S'agissant des transferts de l'Etat aux entreprises, les résultats obtenus s'opposent à ceux de Vartia (2008), OCDE (2009) et Johansson (2011) qui confirment qu'une réorganisation des structures fiscales reposant sur le transfert des recettes fiscales est favorable à la croissance économique. Cela signifie qu'au Congo, les transferts de l'Etat aux entreprises ne sont pas assez bien structurés pour permettre aux entreprises de créer de la richesse, il vaudrait mieux y avoir le moins possible recours.

En ce qui concerne la formation brute de capital fixe, les résultats montrent des effets négatifs sur la croissance économique. Ces résultats coïncident avec les travaux de l'OCDE (2009) et ceux de Poterba (1989) qui trouvent que le capital physique ainsi que les décisions d'investissement et 
d'incitations des entreprises à investir dans des activités novatrices, ont des conséquences négatives sur la productivité et la croissance économique. Dans le cas du Congo, le capital physique ne permet pas d'accroître ni la production des entreprises et ni celle de la nation.

\section{Conclusion}

L'objectif de cet article est d'analyser les impacts des structures fiscales sur la croissance économique au Congo-Brazzaville. Il s'appuie sur les effets des transferts de l'Etat aux entreprises et sur la distinction entre les impôts sur les revenus des personnes physiques, impôts sur les sociétés et impôts sur les biens et services. Les résultats obtenus à partir du modèle de Dickey et Fuller montrent qu'à long terme, les impôts sur les revenus des personnes physiques, les impôts sur les biens et services, les recettes totales de l'Etat, les transferts de l'Etat aux entreprises et la formation brute de capital fixe, favorisent la croissance économique. Tandis qu'à court terme, ce sont les impôts sur les sociétés, les recettes et les transferts de l'Etat qui exercent des effets sur la croissance économique.

En revanche, à long terme, les impôts sur les biens et services et les recettes totales de l'Etat exercent une influence positive sur la croissance. A court terme, seules les recettes totales de l'Etat impactent positivement la croissance. Mais, l'observation des résultats nous montre que les recettes totales de l'Etat influent positivement la croissance économique à long et court terme. Par ailleurs, les transferts de l'Etat aux entreprises impactent négativement la croissance à long et court terme. Cela nous amène à proposer quelques suggestions de politique économique.

La première est relative aux effets des impôts sur les revenus des personnes physiques et des impôts sur les sociétés sur la croissance économique. À priori une augmentation de ces variables a un effet négatif. Donc elles semblent à éviter. Toutefois elles ont un rôle dans la répartition des richesses qui fait qu'on ne peut pas totalement les ignorer.

La deuxième concerne l'impact positif des impôts sur les biens et services sur la croissance. Une augmentation des impôts sur les biens et services augmente la croissance. Si l'Etat veut auto-entretenir celle-ci, il peut dans une certaine mesure jouer sur ce levier.

La troisième se rapporte à l'impact négatif des transferts de l'Etat aux entreprises sur la croissance. Ce résultat suggère que les autorités congolaises doivent limiter ces transferts et revoir les modalités d'attribution pour les restreindre aux seules activités productives.

Comme nous venons de l'observer, les recettes financières de l'Etat ont des effets positifs sur la croissance congolaise, cependant le revenu congolais est tributaire de l'exportation du pétrole. Ainsi, notre dernière recommandation concerne la diversification de l'économie congolaise. 
Comme cette économie repose principalement sur les ressources pétrolières et de l'industrie du bois, compte tenu des turbulences actuelles sur le marché du pétrole, il serait mieux que l'Etat congolais pense à diversifier ses sources de revenus par la valorisation d'autres secteurs de production, tels que l'agriculture, le bois, l'industrie de manufacture naissante, etc.

\section{References:}

1. Adams C S., Bevan D. L. (2005) Fiscal deficits and growth in developing countries, Journal of public economics, 89, 571597.

2. Ades J., Gagné R., Mia H., Lussier J., Martel V., Martin E. (2015) Fiscalité et aide publique aux entreprises : Revue de la littérature et analyse, Montréal, Institut du Québec, 134p.

3. Aghion P., Howitt P. (1988) Growth and cycles through creative destruction, Unpub-lished, University of Western Ontario.

4. Arulampalam W., Devereux M., Maffini G. (2009) The direct incidence of corporate income tax on wages, Oxford university centre for business taxation, WP 09/17.

5. Barro R. J. (1996) Determinants of economic growth: A cross country empirical study. NBER.

6. Bloom N., Sadun R., Reenen J. V. (2007) Americans do I.T. better: US multinationals and the productivity miracle, NBER Working Papers, $\mathrm{n}^{\mathrm{o}} 13085$, National bureau of economic research, Cambridge MA.

7. Bracewell-Milnes B (1976) The camels's back: an international comparison of tax burdens, Center for policy studies, London.

8. Brun J F.,Chambas G., Cambes J. C. (1998) Politique fiscale et croissance économique, Revue d'économie du développement, 2, Juin.

9. Caselli F., Esquivel G., Lefort F. (1996) Reopening the convergence debate: a new look at cross- country growth empirics, Journal of Economic Growth, 1-40.

10. Chambas G. (1994) Fiscalité et développement en Afrique SudSahalienne, Economica.

11. Colm G., Helzner M. (1958) The structure of government revenue and expenditure in relation to the economic development of the U.S., In Brussels Congress of the I.I.F.P.

12. Corlett W.J., Hague D C. (1953) Complementarity and the excess burden of taxation, Review of economic studies, $\mathrm{n}^{\circ} 21$.

13. Cnuced (2014) Le développement économique en Afrique : catalyser l'investissement pour une croissance transformatrice, Rapport, Nations Unies, 122p. 
14. Criscuolo C. (2006) Les filiales de sociétés étrangères dans les économies de l'OCDE: leur poids, leurs performances et leur contribution à la croissance du pays d'accueil, Revue économique de l'OCDE, $\mathrm{n}^{\circ} 41$.

15. DGID (2016), Dispositions fiscales pour la loi de finances 2014, http://www.adiac- congo.com/content/direction-generale-desimpots-des-dispositions-fiscales-pour-la- loi-de-finances-2014.

16. Ebeke C., Ehrhart H. (2010) Tax revenue instability in Sub-Saharan Africa: Consequences and remedies, Working papers, 25, CERDI.

17. Engen E. M., Skinner J. (1996) Taxation and economic growth, National tax Journal, 49(4), 617-642.

18. Fayçal B. A., Saloua B.A. (2016) Impact des recettes fiscales sur la croissance économique: Cas des grands pays et des paradis fiscaux, International Journal of business \& economic strategy, 4, 16.

19. Feldstein M.S. (1974) Tax avoidance and the dead weight loss of the income tax, NBER Working Paper, 5055.

20. Felix R.A. (2006) Passing the burden: Corporate tax incidence in open economics, University of Michigan.

21. FMI (2010) Will it hurt? The macroeconomic effects of fiscal consolidation, dans: World Economic Outlook: Recovery, Risk, and Rebalancing, 93-124.

22. FMI (2014) Questions générales, République du Congo, https://www.imf.org/external/french/pubs/ft/scr/2014/cr14273f.pdf.

23. Gemmel N., Kneller R., Sanz I.(2011) The timing and persistence of fiscal policy impacts on growth: Evidence from OECD countries, Economic Journal 121 (550), 33-58.

24. Gilbert G. (1979) Economie politique des structures fiscales, Thèse de Doctorat, Université Paris X, 580p.

25. Griffith R., Redding S., Simpson H. (2004) Foreign ownership and productivity: New evidence from the services sector and the R\&D Lab, CEP Discussion Paper, $\mathrm{n}^{\circ}$ 649, Centre for Economic.

26. Hasset K.A., Mathur A. (2006) Taxes and wages, American Entreprise Institute, Working Paper, 28.

27. Heady C. (1987) A Diagrammatic approach to optimal commodity taxation, Public finance, 42.

28. Islam N. (1995) Growth empirics: A panel data approach, Quarterly Journal of economics 110 (4), 1127-1170.

29. Johansson Å., Heady C., Arnold J., Brys B., Vartia L. (2008) Fiscalité et croissance économique, Documents de travail du département des affaires économiques, $\mathrm{n}^{\circ} 620$. 
30. Johansson A. (2011) Tax policy for economic recovery and growth, Economic Journal, 121(550), 59-80.

31. Keen M., Mansour M. (2010a) Revenue mobilization in sub-Saharan Africa: Challenges from globalization I-trade reform, Development policy review, 28,552-571.

32. Keller W. (2004) International technology diffusion, Journal of economic literature, 42.

33. Kneller R., Bleaney M.F., Gemmell N. (1999) Fiscal policy and growth: evidence from OECD countries, Journal of public economics, 74, 171-190.

34. Koester J., Reinhard B., Kormendi R.C. (1989) Taxation, aggregate activity and economic growth:cross-country evidence, Journal of publc economics, 80, 604-617.

35. Lakhdar A., El Mustapha K., Habib B. B. (2017) La fiscalité et la croissance économique: une investigation empirique par les modèles ECM, cas du Maroc, Revue Européenne du droit social, XXXVII, Issue 4.

36. Laffer A. (2004) The Laffer Curve: Past, Present, and Future, http ://www.heritage.org/research/ taxes/bg1765.cfm).

37. Levine R., Renelt D. (1992) A sensitivity analysis of cross-country growth regression, American economic review, 82 (4), 942-963.

38. Lucas R. (1988) On the mechanisms of economics development, Journal of monetary economics, 22, 3-42.

39. Mankiw N. G., Romer D., Weil D. N. (1992) Contribution to the empirics of economic growth, Quarterly Journal of economics 107 (2), 407- 437.

40. Mendoza E., Milesi-Ferretti G., Asea P. (1997) On the effectiveness of tax policy 26 in altering long-run growth: Harberger's superneutrality conjecture, Journal of public economics, 66 (1), 99-126.

41. Milesi-Ferretti G. M., Roubini N.(1998) Growth effects of income and consumption taxes, Journal of Money, Credit and banking 30 (4), 721-744.

42. Musgrave R.A. (1969) Fiscal systems, Studies in comparative economics, New haven and London, Yale Univ. Press.

43. Ngakosso A. (2016) La politique fiscale congolaise, DGID,htt://impotsgouv.cg/media/doc/documentation/Congo_Politiqu e_Fiscale.pdf.

44. Ngoc Ha Dao., Arseneau M. (2012) Le dosage des impôts et la croissance économique: des leçons pour le Québec, Université de Sherbrooke, 1-22. 
45. OCDE (2009) Fiscalité et croissance économique, réformes économiques: objectif croissance, $O C D E, 1(5), 228$.

46. Padovano F., Galli E. (2001) Tax rates and economic growth in the OECD Countries (1950- 1990), Economic inquiry , January (39), 44-57.

47. PND Congo (2012-2016) Cadre Macroéconomique et Budgétaire, Livre 3.

48. PNUD Congo (2012) Étude sur la vulnérabilité de l'économie congolaise et ses perspectives de diversification, 228p.

49. Poterba J.M. (1989) Capital gains tax policy towards entrepreneurship, National tax journal, 42.

50. Rawls J. (1971) A theory of justice, Cambridge, Harvard Univ. Press.

51. Romer P.M. (1986) Increasing returns and long-run growth, Journal of political economy, 94, 1002-1037.

52. Romer C.D., Romer D.H. (2010) The macroeconomic effects of tax changes: estimates based on a new measure of fiscal shocks, American economic review, 100 (3), 763-801.

53. Slemrod J. (1990) Optimal Taxation and Optimal Tax Systems, Journal of Economic Perspectives, 4 (1), 157-178.

54. Solow R. (1956) A contribution to the theory of economic growth, Quarterly journal of economics, 65-94.

55. Smith A. (1776) Recherches sur la nature et les causes de la richesse des nations, Traduction française de Germain Garnier, 1881, à partir de l'édition revue par Adolphe Blanqui en 1843.

56. Tanzi V., Zee H. (1997) Fiscal policy and long-run growth, IMF Staff Papers, 44 (2), 179- 209.

57. Vaillancourt F., Pouya E. (2012) L'impact du mix fiscal sur la croissance économique des provinces canadiennes de 1981-2010. Rapport, février, Montréal.

58. Vartia L. (2008) Tax and economic growth, OECD Economics Department Working Papers.

59. Yaya Keho (2010) Effets macroéconomiques de la politique fiscale en Cote d'Ivoire, Politique Economique et Développement $\mathrm{n}^{\circ} 3$, CAPEC.

60. Young L., Gordon R H. (2004) Tax structure and economic growth, Journal of public economics, 89, 1027-1043.

61. Widmalm F. (2001) Tax structure and growth : are some taxes better than others ? public choice, 107,3-4, 199-219. 


\section{Annexes}

Tableau 6 : Résultats du test d'auto corrélation des résidus

Sample: 19812015

Included observations : 35

\begin{tabular}{|c|c|c|c|c|c|c|}
\hline Autocorrelation & Partial Correlation & & $\mathrm{AC}$ & PAC & Q-Stat & Prob \\
\hline$* *$. &.$* 1$. & 1 & -0.174 & -0.174 & 1.1568 & 0.282 \\
\hline .1 & $\cdot 1$ & 2 & 0.042 & 0.012 & 1.2256 & 0.542 \\
\hline. & .1 & 3 & -0.036 & -0.028 & 1.2785 & 0.734 \\
\hline .1. & .1. & 4 & 0.024 & 0.013 & 1.3024 & 0.861 \\
\hline.$* 1$. &.$* 1$. & 5 & -0.080 & -0.075 & 1.5791 & 0.904 \\
\hline$*$. & $*$. & 6 & -0.072 & -0.103 & 1.8087 & 0.936 \\
\hline.$\left.\right|^{*}$. &.$\left.\right|^{*}$. & 7 & 0.141 & 0.121 & 2.7296 & 0.909 \\
\hline .1 & .1 & 8 & -0.060 & -0.017 & 2.9008 & 0.940 \\
\hline .1 &. & 9 & -0.035 & -0.061 & 2.9611 & 0.966 \\
\hline$* \mid$ & $* 1$ & 10 & -0.089 & -0.106 & 3.3712 & 0.971 \\
\hline$\left.\right|^{* *}$ & | $\left.\right|^{* *}$ & 11 & 0.348 & 0.325 & 9.9053 & 0.539 \\
\hline .*. & .1. & 12 & -0.147 & -0.032 & 11.128 & 0.518 \\
\hline & 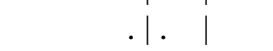 & 13 & 0.027 & -0.023 & 11.170 & 0.597 \\
\hline & .1 & 14 & 0.022 & 0.018 & 11.200 & 0.670 \\
\hline & $*$ & 15 & -0.061 & -0.072 & 11.445 & 0.720 \\
\hline.$*$ & **1 & 16 & -0.130 & -0.117 & 12.595 & 0.702 \\
\hline
\end{tabular}

Tableau 7 : Résultats du test de Hétéroscédasticité de Breusch-Pagan-Godfrey

\begin{tabular}{|cccc|}
\hline F-statistic & 0.765822 & Prob. F(7,27) & 0.6204 \\
Obs*R-squared & 5.797963 & Prob. Chi-Square(7) & 0.5635 \\
Scaled explained SS & 6.487631 & Prob. Chi-Square(7) & 0.4841 \\
\hline
\end{tabular}

